\title{
Engineering the anthocyanin regulatory complex of strawberry (Fragaria vesca)
}

\section{Kui Lin-Wang ${ }^{1}$, Tony K. McGhie ${ }^{2}$, Mindy Wang ${ }^{1}$, Yuhui Liu ${ }^{3}$, Benjamin Warren ${ }^{1}$, Roy Storey ${ }^{4}$, Richard V. Espley ${ }^{1 *}$ and Andrew C. Allan ${ }^{1,5 *}$}

${ }^{1}$ The New Zealand Institute for Plant and Food Research Limited, Auckland, New Zealand

2 Plant and Food Research Limited, Palmerston North, New Zealand

${ }^{3}$ Gansu Key Lab of Crop Improvement and Germplasm Enhancement, Gansu Agricultural University, Lanzhou, China

${ }^{4}$ Plant and Food Research Limited, Te Puke, New Zealand

${ }^{5}$ School of Biological Sciences, University of Auckland, Auckland, New Zealand

\section{Edited by:}

Stefan Martens, Edmund Mach

Foundation, Italy

Reviewed by:

Kathrin Schrick, Kansas State

University, USA

Paula Elomaa, University of Helsinki,

Finland

\section{*Correspondence:}

Richard V. Espley, The New Zealand

Institute for Plant and Food

Research Limited, 120 Mount Albert

Road, Private Bag 92169, Auckland

1025, New Zealand

e-mail: richard.espley@

plantandfood.co.nz;

Andrew C. Allan, The New Zealand

Institute for Plant and Food

Research Limited, 120 Mount Albert

Road, Private Bag 92169, Auckland

1025, New Zealand; School of

Biological Sciences, University of

Auckland, Biology Building, 5

Symonds Street, Private Bag 92019,

Auckland 1010, New Zealand

e-mail: andrew.allan@

plantandfood.co.nz
The woodland strawberry, Fragaria vesca is a model fruit for a number of rosaceous crops. We have engineered altered concentrations of anthocyanin in $F$. vesca, to determine the impact on plant growth and fruit quality. Anthocyanin concentrations were significantly increased by over-expression or decreased by knock-down of the R2R3 MYB activator, MYB10. In contrast, a potential bHLH partner for MYB10 (bHLH33) did not affect the anthocyanin pathway when knocked down using RNAi constructs. Metabolic analysis of fruits revealed that, of all the polyphenolics surveyed, only cyanidin, and pelargonidin glucoside, and coumaryl hexose were significantly affected by over-expression and knock down of MYB10. Using the F. vesca genome sequence, members of the MYB, bHLH, and WD40 families were examined. Global analysis of gene expression and targeted qPCR analysis of biosynthetic genes and regulators confirmed the effects of altering MYB10 expression, as well as the knock-down of bHLH33. Other members of the MYB transcription factor family were affected by the transgenes. Transient expression of strawberry genes in Nicotiana benthamiana revealed that MYB10 can auto-regulate itself, and potential repressors of MYB10. In tobacco, MYB10's activation of biosynthetic steps is inhibited by the strawberry repressor MYB1.

Keywords: Fragaria vesca, FvMYB10, transcription factor, regulation, anthocyanin, strawberry

\section{INTRODUCTION}

The woodland strawberry, Fragaria vesca $(2 \mathrm{n}=2 \mathrm{x}=14)$, has a small genome (240 Mb) (Folta and Davis, 2006), and a short generation time. It is easy to propagate both from seed and clonally, and can be efficiently transformed by Agrobacterium-mediated transformation (Oosumi et al., 2006) to enhance secondary plant products (Bulley et al., 2012). Therefore, it has become a model plant for the diverse Rosaceae family, which contains many valuable fruit and ornamental crops (Folta and Dhingra, 2006).

Strawberry fruits are distinguished by anthocyanins, the watersoluble flavonoid pigments that are found in higher plants and are responsible for the red, purple, and blue color of many fruits, flowers, leaves and seeds. There is growing evidence from epidemiological, in vitro and in vivo studies for the role of anthocyanins in potential long-term health benefits and an association with reduced incidence of chronic diseases, although there are still many questions regarding the mechanistic action and specific health effects (Traka and Mithen, 2011). Anthocyanins have been the target of a number of recent studies concerning the improvement of the dietary health benefits of fruit (Davies and
Espley, 2013). Intervention trials using highly anthocyanic maize, tomato, and apple have all shown a number of positive health effects in small mammals (Butelli et al., 2008; Toufektsian et al., 2008; Espley et al., 2014). The evidence makes a strong case for further investigation and the development of foods with high concentrations of bioactive secondary metabolites, regardless of the breeding approach employed. One method might be the manipulation of secondary metabolite pathway regulation.

In all species studied to date, the regulation of anthocyanin biosynthesis is at the level of transcriptional regulation of genes encoding enzymes of the biosynthetic steps by transcription factors that include MYB, bHLH, and WD-repeat proteins (the MYB-bHLH-WD40 "MBW" complex, Baudry et al., 2006; Allan et al., 2008; Butelli et al., 2008; Jaakola, 2013). MYB genes are identified by their conserved DNA-binding domain consisting of one or more repeats and those that control anthocyanin are highly conserved amongst many species examined and fall into a specific subgroup (Allan et al., 2008). The MYB genes involved in the "MBW" complex are the two repeat R2R3 class. There are both activating and repressing R2R3 MYBs. Over-expression 
of the MYB member of the complex has dramatic effects. In Arabidopsis, over-expression of AtPAP1 or AtPAP2 results in the accumulation of anthocyanin (Gonzalez et al., 2008). The concentration of anthocyanin is substantially elevated by over-expressing both bHLH (Del) and MYB (Ros1) genes from the snapdragon Antirrhinum majus in the fruit of transgenic tomatoes (Butelli et al., 2008). In one of these transgenic tomato lines the highest concentration of $2.83 \pm 0.46 \mathrm{mg}$ of anthocyanin per $\mathrm{g}$ fresh weight was measured, while anthocyanins were virtually undetectable in wild-type tomato fruit. In apple, overexpression of MdMYB10 in "Royal Gala," a white-fleshed, green-leaved apple cultivar results in a red leaf and fruit phenotype (Espley et al., 2007). Furthermore, environmental factors (i.e., temperature and light) can alter the red coloration of Arabidopsis over-expressing AtPAP1 plants (Rowan et al., 2009). High temperatures $\left(30-37^{\circ} \mathrm{C}\right)$ have been shown to decrease anthocyanin content in the skin of apple (Lin-Wang et al., 2011).

Repressors of anthocyanin accumulation were also identified within the MYB family of transcription factors, including AtMYB3, AtMYB4, and AtMYBL2 from Arabidopsis (Jin et al., 2000; Dubos et al., 2008), MdMYB16, MdMYB17, and MdMYB111 from apple (Lin-Wang et al., 2011), and FaMYB1 and FcMYB1 from strawberry (Aharoni et al., 2001; Salvatierra et al., 2013). It was reported that flowers of transgenic tobacco lines overexpressing FaMYB1 showed a severe reduction in pigmentation, and a reduction in the concentration of cyanidin 3rutinoside (an anthocyanin) and quercetin-glycosides (flavonols) was detected (Aharoni et al., 2001). Another MYB family member FaMYB5 was also described as a putative negative regulator in the strawberry proanthocyanidin biosynthesis pathway (Schaart et al., 2013).

In the Fragaria genus, fruit color is determined by the accumulation of anthocyanin, the most abundant flavonoid-derived component in strawberry fruits (Hannum, 2004). Over-expression of the anthocyanin biosynthesis activator FaMYB10 in F. ananassa results in plants with elevated root, foliar, and fruit anthocyanin concentrations (Lin-Wang et al., 2010). A recent study showed that over-expression of Arabidopsis proanthocyanidin (PA) biosynthesis regulators AtTT2, AtTT8, and AtTTG1 in F. ananassa resulted in a partial loss of red coloration in the flesh, associated with an increased PA content and decreased anthocyanin accumulation (Schaart et al., 2013). In contrast, the silencing of the PA biosynthesis enzyme anthocyanidin reductase in strawberry resulted in a redirection of flavonoid production away from PAs and an increase in anthocyanin production during early fruit development (Fischer et al., 2014) whilst silencing FaGT1, the 3-0-glucosyltransferase implicated in glycosylating anthocyanidins, has been shown to specifically reduce anthocyanins with a concomitant increase in flavan-3-ols (Griesser et al., 2008).

In this study we used the diploid strawberry F. Vesca as a model for the related octoploid cultivated strawberry, F. ananassa. $F$. Vesca benefits from a relatively small genome, good transformation efficiency, and ease of propagation. We describe how overexpression of $F v M Y B 10$ in F. vesca produced purple-skinned and red-fleshed strawberries, while knock down of FvMYB10, using an RNAi construct produced white-skinned and white-fleshed fruit. The phenotypic, metabolomic and expression data for the stable transgenic lines 35S:FvMYB10 and FvMYB10 RNAi confirmed that $F v M Y B 10$ plays a key role in activating anthocyanin biosynthesis pathway in $F$. vesca. Stable transformation in this model rosaceous species provides information on plant performance in the presence of altered levels of anthocyanins and suggests the possibility of developing novel strawberry crops by traditional or non-traditional breeding technologies. This information can further be translated to other rosaceous fruit crops to help to understand the effect manipulating metabolite levels.

\section{MATERIALS AND METHODS STRAWBERRY TRANSFORMATION}

"Alpine" strawberry F. vesca ssp. vesca was selected for gene isolation and plant transformation. For overexpression of FvMYB10, the full-length cDNA of FvMYB10 was cloned into the pSAK277 vector (Gleave, 1992). For knock down expression of FvMYB10 and FvbHLH33, cDNA fragments corresponding to nucleotides 171-636 and 609-1088 of FvMYB10 and FvbHLH33, respectively (relative to the ATG start codon) were selected. Sequences were blasted to the strawberry genome (http://www.rosaceae.org/tools/ncbi_blast) to confirm the specificity to the gene of interest only. The FvMYB10 fragment was cloned using the $5^{\prime}$ to $3^{\prime}$ primer sequences GCCGAATATCAAGAGAGGAGAGTTT (forward) and GAAGTCATGATCCTCCAAACCAATA (reverse). The $F v b H L H$ fragment was cloned using the $5^{\prime}$ to $3^{\prime}$ primer sequences AAAGCCAGATTGCTCCGAGAAA (forward) and AGACCCAAGGAGCTGAACTTTGTAT (reverse). The fragments were cloned into a commercial vector $\mathrm{pENTR/D-TOPO} \mathrm{(pENTR}$ Directional TOPO Cloning Kits, Life Technologies) respectively. Two copies of the selected gene fragment were inserted into an RNAi vector pTKO2 in an inverse orientation, flanking an intron sequence, using a Gateway LR reaction (Snowden et al., 2005). The construct was electroporated into Agrobacterium tumefaciens GV3101, then transformed into F. vesca, using the Agrobacterium-mediated strawberry transformation protocol as previously described (Oosumi et al., 2006).

\section{LIQUID CHROMATOGRAPHY-QUADRUPOLE-TIME OF FLIGHT-MASS SPECTROMETRY ANALYSIS OF ANTHOCYANINS}

Samples of frozen ground fruit, approximately $150 \mathrm{mg}$, were extracted overnight at $1^{\circ} \mathrm{C}$ with $1.0 \mathrm{~mL}$ solvent (ethanol/water/formic acid 80/20/1 v/v/v). The samples were centrifuged, diluted and analyzed by liquid chromatography and quadrupole time of flight, high resolution, mass spectrometry (LC-QTOF-HRMS). The LC-MS system was composed of a Dionex Ultimate ${ }^{\circledR} 3000$ Rapid Separation LC and a micrOTOF QII high resolution mass spectrometer (Bruker Daltonics, Bremen, Germany) fitted with an electrospray ion source. Component separation by LC was achieved using two UHPLC columns connected in series. The columns were both Zorbax $^{\mathrm{TM}}$ SB-C18 $2.1 \times 100 \mathrm{~mm}, 1.8 \mu \mathrm{m}$ (Agilent, Melbourne, VIC, Australia) and were maintained at $60^{\circ} \mathrm{C}$. The flow was $350 \mu \mathrm{L} / \mathrm{min}$. The solvents were $\mathrm{A}=100 \%$ acetronitrile, and $\mathrm{B}=0.4 \%$ formic acid. The solvent gradient was: $10 \% \mathrm{~A}, 90 \% \mathrm{~B}$, 0-0.5 min; linear gradient to $100 \% \mathrm{~A}, 0.5-25 \mathrm{~min}$; composition held at $100 \%$ A, 25-28 min; linear gradient to $10 \% \mathrm{~A}, 90 \% \mathrm{~B}$, 28-28.2 $\mathrm{min}$; to return to the initial conditions before another 
sample injection at $31 \mathrm{~min}$. The injection volume for samples and standards was $1 \mu \mathrm{L}$. The micrOTOF QII parameters for polyphenolic analysis were: temperature $225^{\circ} \mathrm{C}$; drying $\mathrm{N}_{2}$ flow $6 \mathrm{~L} / \mathrm{min}$; nebulizer $\mathrm{N}_{2} 1.5$ bar, endplate offset $-500 \mathrm{~V}$, mass range $100-1500 \mathrm{Da}$, acquired were acquired at $2 \mathrm{scans} / \mathrm{s}$. Negative ion electrospray was used with a capillary voltage of $+3500 \mathrm{~V}$. Post-acquisition internal mass calibration used sodium formate clusters, with the sodium formate delivered by a syringe pump at the start of each chromatographic analysis. Polyphenols compounds were quantified using QuantAnalysis (Bruker Daltonics, Bremen, Germany) software. To quantify target compounds, exact $( \pm 10 \mathrm{mDa})$ ion chromatograms (EICs) for each of the target compounds were extracted from the three dimensional LCMS data of each sample and calibration standard. The concentrations of components in samples were calculated by comparison with external calibration curves of authentic compounds. When an authentic compound was not available, the calibration curve of a similar compound was used to calculate equivalents. For example all anthocyanins were quantified as cyanidin 3-glucoside equivalents.

\section{REAL-TIME qPCR EXPRESSION ANALYSIS}

Mature strawberry fruit were collected from the over-expressing lines, RNAi lines and wild-type controls. Total RNA from three biological replicates per time point was extracted, using a method adapted from Chang et al. (1993). Removal of genomic DNA contamination and first-strand cDNA synthesis were carried out using oligo (dT) according to the manufacturer's instructions (QuantiTect Reverse Transcription Kit, Qiagen). Realtime qPCR DNA amplification and analysis was carried out using the LightCycler 480 Real-Time PCR System (Roche), with LightCycler 480 software version 1.5. The LightCycler 480 SYBR Green I Master Mix (Roche) was used following the manufacturer's method. The qPCR conditions were $5 \mathrm{~min}$ at $95^{\circ} \mathrm{C}$, followed by 45 cycles of $5 \mathrm{~s}$ at $95^{\circ} \mathrm{C}, 5 \mathrm{~s}$ at $60^{\circ} \mathrm{C}$, and $10 \mathrm{~s}$ at $72^{\circ} \mathrm{C}$, followed by $65-95^{\circ} \mathrm{C}$ melting curve detection. The qPCR efficiency of each gene was obtained by analyzing the standard curve of a cDNA serial dilution of that gene. Five reference genes ACTIN (XM_004307470), GAPDH (XM_004309993), PP2a (XM_004297079), SAND (gene21567-v1.0-hybrid, Genome Database for Rosacea), and UBC9 (XM_004307970) were examined. GAPDH (XM_004309993), PP2a (XM_004297079), and UBC9 (XM_004307970) were selected because of their consistent transcript levels throughout samples, with crossing threshold values changing by less than 2 . Transcript abundance was calculated relative to the geometric mean of GAPDH, $P P 2 a$, and UBC9 (Pfaffl, 2001). All primer sequences are listed in Supplementary Table 1.

\section{PHYLOGENETIC ANALYSIS}

The candidate genes FvMYB1, FvMYB5, FvbHLH3, FvbHLH33, FvMYC1, and FvTTG1 were selected by blasting protein sequences of published corresponding genes in $F$. ananassa to "Non-redundant protein sequences (nr)" in NCBI protein database, and then taking the highest-scoring genes (in our case, $E$ values were less than $8 \mathrm{e}-126)$ and then performing a reciprocal blast back to the NCBI protein database to confirm the original genes had the highest scores. Protein sequences were aligned using Blosum62 (Gap open penalty: 90 and Gap extension penalty: 3) in Geneious 6.1.7. Phylogenetic trees were generated using MEGA 6.06 and the Neighbor-Joining method with 1000 bootstrap replicates.

\section{DUAL LUCIFERASE ASSAY OF TRANSIENTLY TRANSFORMED NICOTIANA BENTHAMIANA LEAVES}

A fragment containing around $1500 \mathrm{bp}$ upstream of the start code ATG of each candidate gene was isolated by PCR, and inserted into the cloning site of pGreenII 0800-LUC (Hellens et al., 2005), and modified to introduce an NcoI site at the $3^{\prime}$ end of the sequence. This allowed the promoter to be cloned as a transcriptional fusion with the firefly luciferase gene (LUC). The promoter-LUC fusion in pGreenII 0800-LUC was used in transient transformation by mixing $100 \mu \mathrm{l}$ of Agrobacterium strain GV3101 (MP90) transformed with the reporter cassette with $300 \mu \mathrm{l}$ each of three other Agrobacterium cultures. These three cultures had been transformed with cassettes containing a cDNA of MYB TF gene or a bHLH TF gene fused to the $35 \mathrm{~S}$ promoter, respectively, in either pSAK277 (Gleave, 1992), or pHex2 (Hellens et al., 2005). N. benthamiana growing conditions, Agrobacterium infiltration processes and luminescent measurements were as described by Hellens et al. (2005).

\section{DEEP SEQUENCING AND TRANSCRIPTOME ANALYSIS}

Four RNA-seq libraries from the pooled mature fruit of 35S:FvMYB10-2, FvMYB10RNAi-2, FvbHLH33RNAi-1, and WT4 were prepared from total RNA using a method adapted from Chang et al. (1993). cDNA library construction from total RNA and next generation sequencing (NGS) were carried out at Macrogen, Inc., Korea. HiSeq2000 platform was implemented and the libraries were sequenced as $100 \mathrm{bp}$ pairedend reads. Around 70 million reads were generated from each library. Deep sequencing reads were quality assessed with the quality assessment software FastQC (Blankenberg et al., 2010). The adaptor sequences were removed for all the reads. The reads were trimmed ( $15 \mathrm{bp}$ from the $5^{\prime}$ end, and $5 \mathrm{bp}$ from the $3^{\prime}$ end) and quality filtered (quality cut-off value: 20 , percentage of bases in sequence that must have quality equal to/higher than cut-off value: 90 ). The reads were mapped to the reference "fvesca_v1.0_genemark_hybrid.annotated.fna" (downloaded from Genome Database for Rosaceae, www.rosaceae.org) with BWA (Li and Durbin, 2009). Over 60\% reads were successfully mapped to the reference. RPKM (reads per kilobase per million mapped reads) was estimated with Cufflinks (Trapnell et al., 2010). Hierarchical cluster analysis of FvMYBs expression based on normalized RPKM value was plotted using a custom $R$ script.

\section{HEADSPACE VOLATILE ANALYSIS BY GC-MS}

Mature strawberry fruit were harvested from green house at 10 to 11 am during November to December 2013. For each of three replicates, about 20 grams of the fruit (more than 20 strawberries) was weighed in a $250 \mathrm{~mL}$ conical flask. Headspace volatiles from the flask were collected with the purge and trap method according to Wang et al. (2011). Volatiles above the fruit were blown 


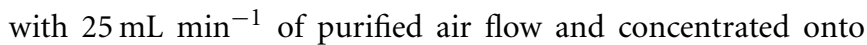
an absorbent column packed with $80 \mathrm{mg}$ Tenax ${ }^{T A} 60 / 80$ (Alltech) attached to an outlet of the system. Sample collection time was $3 \mathrm{~h}$ at room temperature. Ethyl ester $(0.2 \mathrm{~mL})$ was added to the top of the absorbent column three times. Eluate $(0.5 \mathrm{~mL})$ was collected from the column at a low pressure with a pre-weighed $1.5 \mathrm{~mL}$ auto-GC injection vial. The ether extract elute was weighed and $2 \mu \mathrm{L}$ of $0.9 \mu \mathrm{g} \mu \mathrm{L}^{-1}$ of hexadecane was added as the internal standard before GC-MS analysis. Ether elute was analyzed with GC-MS (7890A-5975C, Agilent Technologies) with a 50:1 split ratio. One $\mu \mathrm{L}$ of the ether elute was injected into the $\mathrm{GC}$ at $250^{\circ} \mathrm{C}$ (Column: Restek Rxi-5 ms, $30 \mathrm{~m} \times 250 \mu \mathrm{m} \times 0.25 \mu \mathrm{m}$ ). The oven temperature was $60^{\circ} \mathrm{C}$ for $2 \mathrm{~min}$; then $6^{\circ} \mathrm{C} \mathrm{min}{ }^{-1}$ to $120^{\circ} \mathrm{C} ; 10^{\circ} \mathrm{C} \mathrm{min}^{-1}$ to $200^{\circ} \mathrm{C} ; 30^{\circ} \mathrm{C} \mathrm{min}^{-1}$ to $250^{\circ} \mathrm{C} ; 60^{\circ} \mathrm{C} \mathrm{min}^{-1}$ to $300^{\circ} \mathrm{C}$; then hold $300^{\circ} \mathrm{C}$ for $3 \mathrm{~min}$. MS acquisition scan mode was set with low mass $=35$ and high mass $=400$. EM voltage was 1212 volts. MS source temperature was $230^{\circ} \mathrm{C}$. Peaks from the sample were integrated with MSD ChemStation E02.02.1431. The mass spectrometry trail of each peak was identified by comparing with the standard spectra recorded in the Nist05a library or the in-house authentic standards and assisted with compound Kovats retention index. The amount of each compound in the fruit ( $\mu \mathrm{g} \mathrm{g}^{-1}$ of the fresh fruit) was calculated with the standard curves prepared with a standard solution with known deferent concentration of ethyl butanoate, ethyl hexanoate, alpha pinene, and beta-myrcene prepared for strawberry fruit.

\section{STATISTICS}

For qPCR analysis, data are presented as means $( \pm S E)$ of three biological replicates. For transient transformation promoter activation assays, data are presented as means $( \pm S E)$ of four biological replicates. For the analysis of anthocyanins, polyphenols and volatile compounds, data are presented as means $( \pm S E)$ of three biological replicates. Statistical significance was determined by one-way ANOVA.

\section{RESULTS \\ GENERATION OF 35S:FvMYB10, FvMYB10 RNAi, AND FvBHLH33 RNAi TRANSGENIC STRAWBERRY LINES}

F. vesca was transformed with a construct containing 35S:FvMYB10. Leaves, petioles, stigmas, and petals of the 35S:FvMYB10 lines were pigmented, and mature fruit of these lines had dark red/purple skin and red flesh, compared with red skin and white flesh in wild-type mature fruit (Figure 1). All the 35S:FvMYB10 lines showed the red phenotype, although the degree of red coloration varied among the four transgenic lines. A previous study showed that when 35S:FaMYB10 was transformed into $F$. ananassa, plants were not highly pigmented, although mature fruit from 35S:FaMYB10 lines showed approximately $50 \%$ more anthocyanin than wild-type controls (Lin-Wang et al., 2010). The transcript level of FvMYB10 expression in the four 35S:FvMYB10 lines were elevated by 6 to 30 -fold compared with that in wild-type controls (Figure 2).

The mature fruit of FvMYB10 RNAi lines had white skin and white flesh and the only pigmented tissue in these RNAi lines was the petiole (Figure 1). Four FvMYB10 RNAi lines were generated in this study, with only two lines (MYB10 RNAi-2 and MYB10

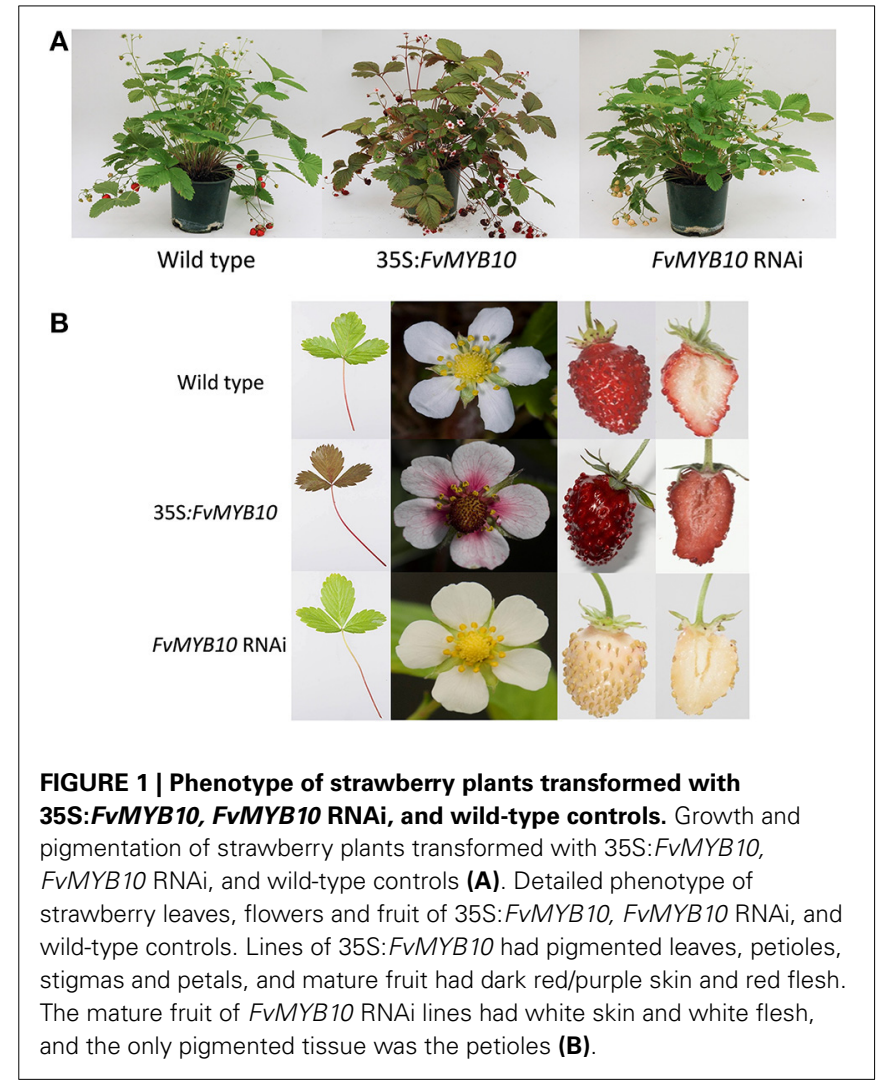

RNAi-4) having white fruit and two lines (MYB10 RNAi-1 and MYB10 RNAi-3) showed pigmented fruit comparable to wild type (WT) (not shown in figures). In contrast, the mature fruit of two lines of FvbHLH33 RNAi lines showed unaltered pigmentation compared to WT (not shown in figures). To determine the efficiency of the RNAi knock-down constructs, quantitative realtime PCR (qPCR) was performed on all lines (Figure 2). This shows that in the white-fruited lines (MYB10 RNAi-2 and MYB10 RNAi-4) there was the lowest expression of $M Y B 10$. This also correlated with a slight increase in $b H L H 33$ transcript. Although the FvbHLH33 RNAi lines showed unaltered pigmentation phenotype compared to WT, the transcript of $b H L H 33$ was significantly reduced.

\section{LIQUID CHROMATOGRAPHY-QUADRUPOLE-TIME OF FLIGHT-MASS SPECTROMETRY ANALYSIS OF ANTHOCYANINS AND POLYPHENOLS}

Pigmentation of the mature fruit in most strawberry cultivars (F. ananassa) is mainly due to the accumulation of pelargonidin glucoside and a small fraction of cyanidin glucoside (Fait et al., 2008). In this study, we used LC-MS to show that anthocyanin in the mature fruit of wild strawberry (F. vesca) is largely due to the accumulation of both cyanidin glucoside and pelargonidin glucoside and a small fraction of pelargonidin malonylglucoside (Figure 3A). There were large increases in both cyanidin glucoside and pelargonidin glusoside (2 to 5-fold) in the 35S:FvMYB10 over-expressing lines. These two compounds were almost undetectable in two FvMYB10 RNAi knock-down 


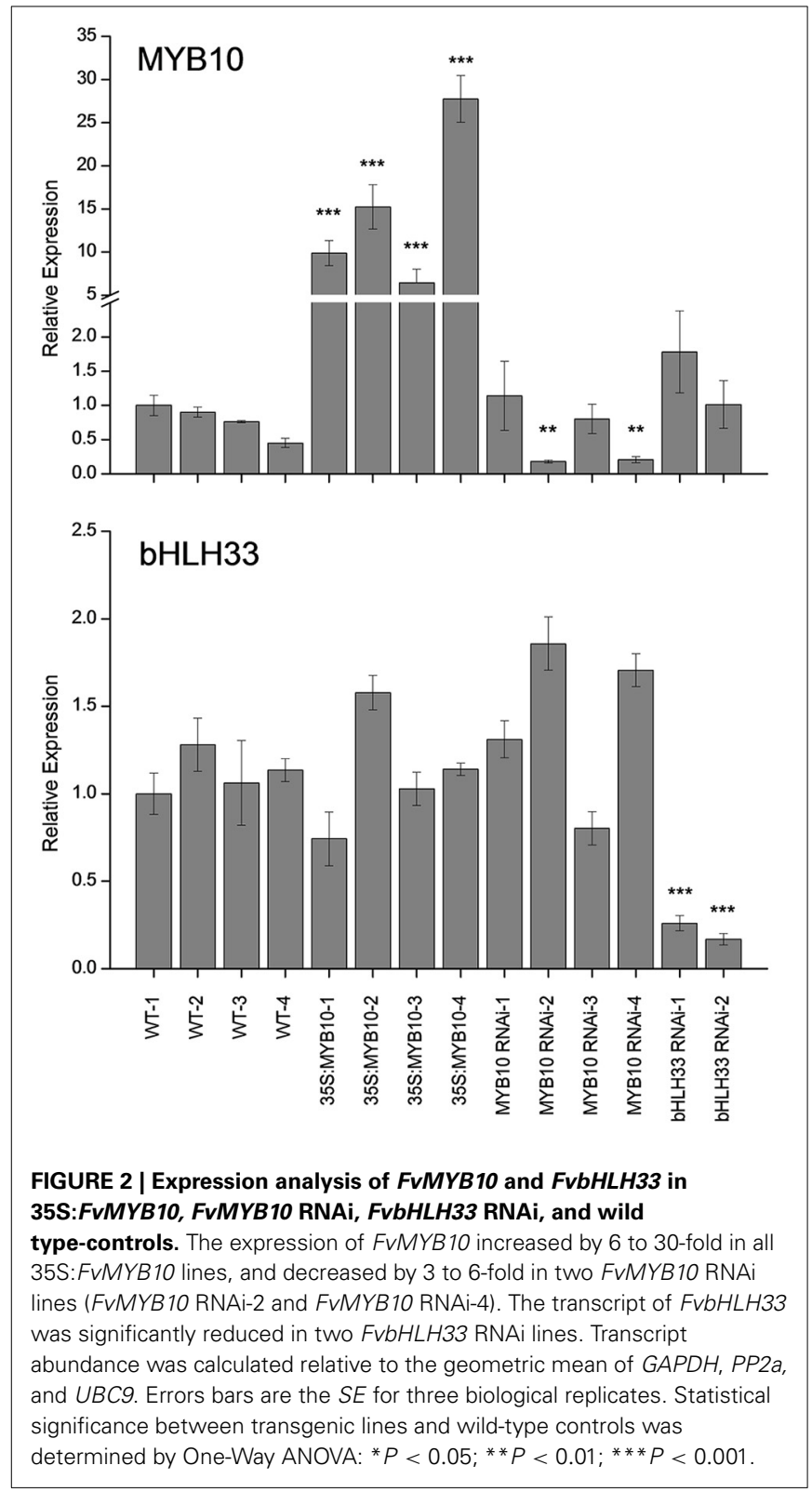

lines (FvMYB10 RNAi-2 and FvMYB10 RNAi-4) which at maturity had white fruit. The concentrations stayed at a similar level to that in wild-type controls in the other two FvMYB10 RNAi knock-down lines (FvMYB10 RNAi-1 and FvMYB10 RNAi-3) which showed unaltered pigmentation when compared to WT (Figure 3A). The accumulation of anthocyanin was not affected by FvbHLH33 RNAi knock-down. There were no significant changes in pelargonidin malonyl glucoside across all the transgenic lines and wild-type controls. However, the amount of this compound detected in the mature fruit was much lower than amounts of the other two compounds.

The LC-MS data for other polyphenols are shown in Supplementary Figure 1 and Supplementary Table 2. Of all the compounds detected, only p-coumaryl glucose (Cou-hex) had a similar concentration profile to that of the two main anthocyanin compounds (cyanidin glucoside and pelargonidin
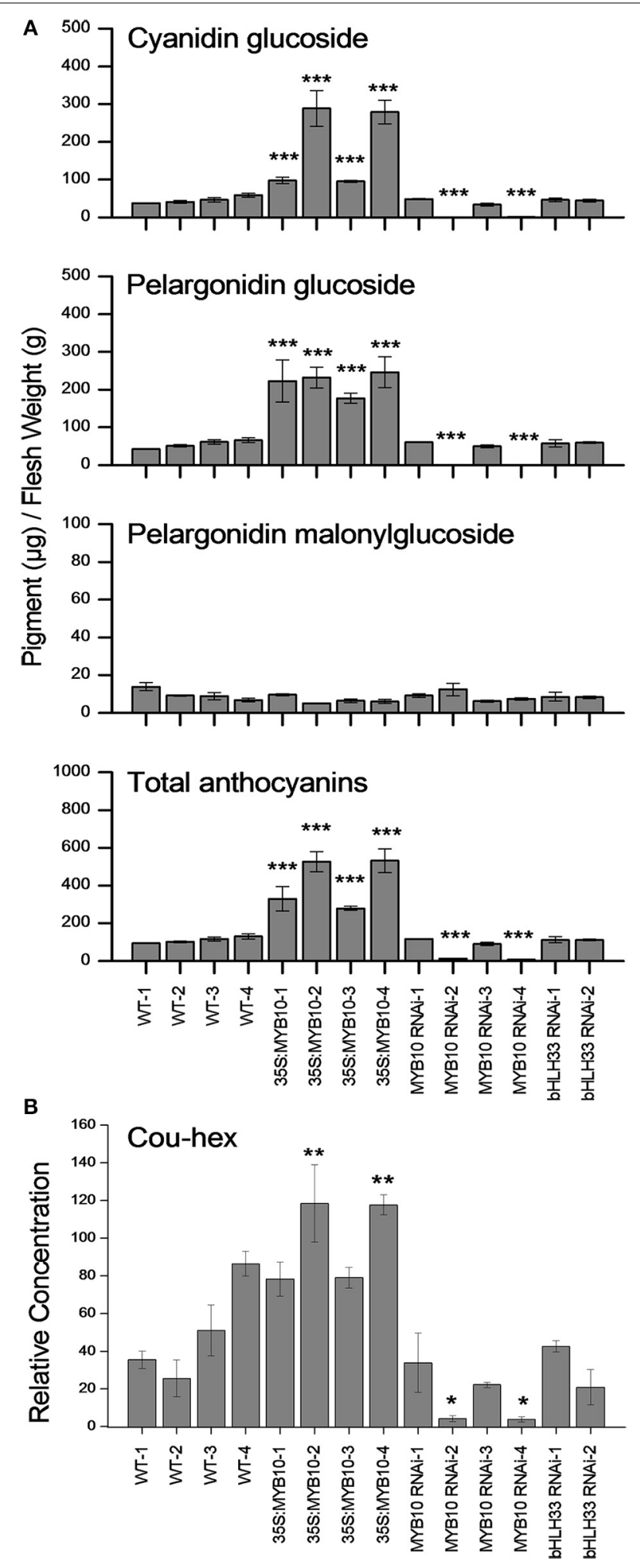

FIGURE 3 | Concentrations of anthocyanins and p-coumaryl glucose in the mature fruit of 35S:FvMYB10, FvMYB10 RNAi, FvbHLH33 RNAi, and wild-type controls. The two main anthocyanins, cyanidin glucoside and pelargonidin glucoside, were elevated in 35S:FVMYB10 lines and barely detectable in two FVMYB10 RNAi lines. The third anthocyanin compound,

(Continued) 


\section{FIGURE 3 | Continued}

pelargonidin malonylglucoside, remained at low concentrations in all fruit. There was no significant difference in anthocyanin concentration between FvbHLH33 RNAi lines and wild-type controls (A). P-coumaryl glucose (Cou-hex) correlated with two main anthocyanin compounds (cyanidin glucoside and pelargonidin glusoside) (B). Errors bars are the SE for three biological replicates. Statistical significance between transgenic lines and wild-type controls was determined by One-Way ANOVA: ${ }^{*} P<0.05$; ${ }^{*} P<0.01 ;{ }^{*}{ }^{*} P<0.001$

glusoside) (Figure 3B). A similar effect on p-coumaryl glucose was seen in $F$. ananassa fruit when FaMYB10 was transiently knocked-down (Medina-Puche et al., 2014).

\section{PHYLOGENETIC ANALYSIS OF HOMOLOGOUS TRANSCRIPTIONAL REGULATORS IN ANTHOCYANIN BIOSYNTHETIC PATHWAY}

To examine potential redundancy in the genes targeted for transformation of $F$. vesca, phylogenetic relationships were determined [using MEGA 5.2 and the neighbor-joining method and with 1000 bootstrap replicates (Tamura et al., 2011)] between Fragaria anthocyanin-related MYB, bHLH and WD40 repeat regulatory proteins and the known homologous transcription factor (TF) genes from Arabidopsis, Malus x domestica, Petunia x hybrida, Zea mays, and Vitis vinifera (Figure 4). All the MYBs associated with promoting anthocyanin biosynthesis clustered within the same subgroup as Arabidopsis PAP1, while MYB repressors clustered outside this subgroup (Figure 4A). All the bHLH genes were divided into two subgroups: one represented by Arabidopsis EGL3-like bHLHs and another by Arabidopsis TT8-like bHLHs (Figure 4B). There was only one AtTTG1-like WD repeat gene selected from each selected species, and the WD repeat genes from dicots clustered together into one subgroup, while the monocot $\mathrm{ZmPAC1}$ was located outside this subgroup (Figure 4C).

\section{ANALYSIS OF GLOBAL GENE EXPRESSION REVEALS THE GENES MOST AFFECTED BY MANIPULATION OF MYB10 LEVELS}

To ascertain the differentially expressed genes in transformed F. vesca fruits, four RNA-seq libraries from mature fruit of 35S:FvMYB10-2, FvMYB10RNAi-2, FvbHLH33RNAi-1, and wild type were prepared and sequenced. Over 290 million reads were generated. Analysis of differential expression in these libraries revealed that the transcript levels of 480 genes in 35S:FvMYB10 lines were greater than two-fold different than those in the wild type, while the transcript levels of 1126 genes were reduced by greater than two fold (Supplementary Tables 3,4 ). In the FvMYB10RNAi lines 1102 genes showed a higher level of transcript abundance greater than two fold, while 2243 genes were reduced by greater than two fold (Supplementary Tables 5, 6). In all these analyses, a cut-off value of greater than or equal to 10 reads per kilobase per million reads (RPKM) was applied.

A relatively simple comparison was made of these long RNAseq lists; an analysis of the intersection between the genes that were more than two-fold up-regulated in 35S:FvMYB10 (in comparison with wild-type fruit), and those that were also more than two-fold down-regulated in FvMYB10 RNAi-2 (in comparison with wild-type fruit) (Table 1). Of particular note was the gene

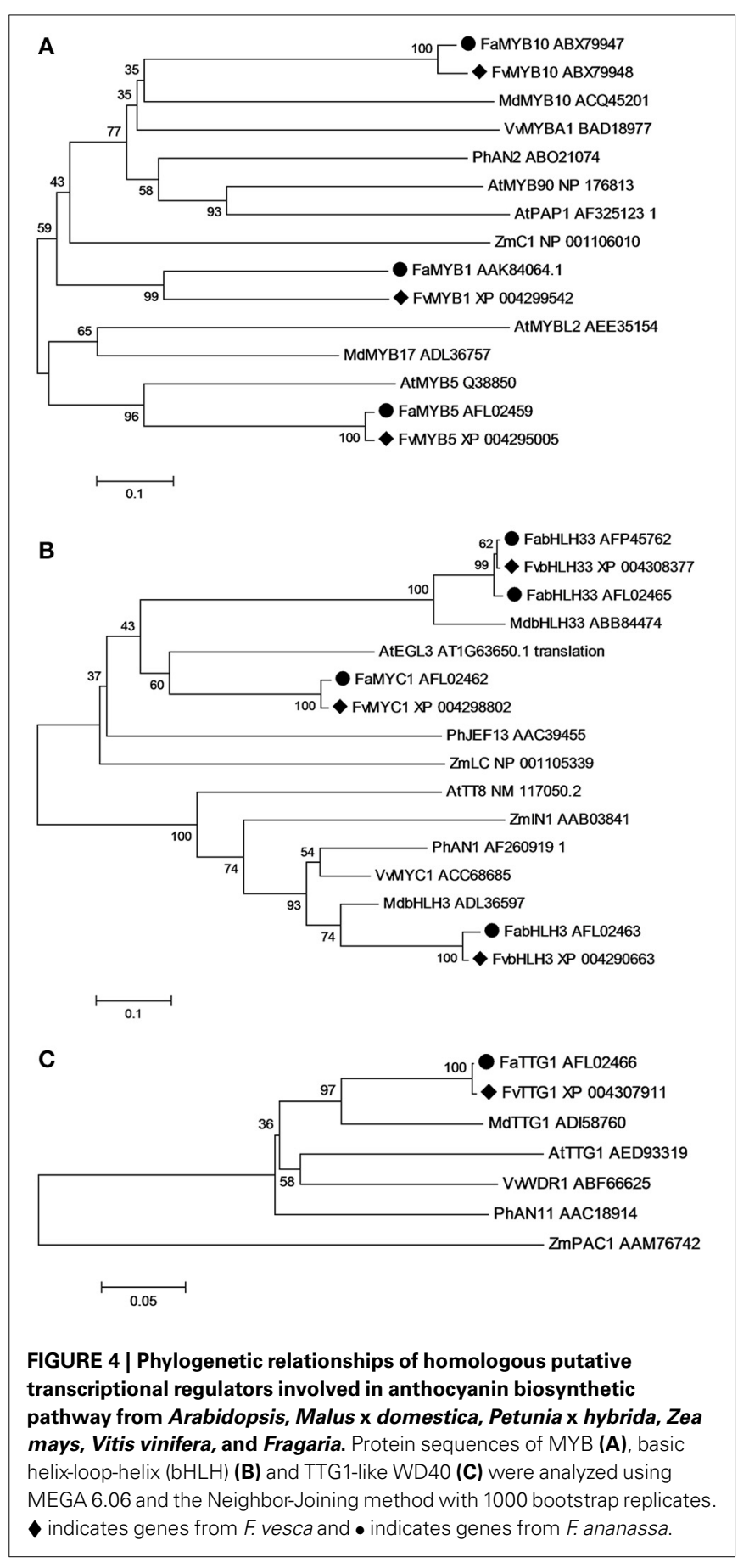

most altered in its expression level, a GST with sequence homology to TT19 of Arabidopsis (54\% identical at the amino acid level to TT19, AT5G17220; although 56\% identical to AT3G03190, AtGST6). TT19 functions as a carrier-transporter for cyanidin3-O-glycoside in Arabidopsis (Sun et al., 2012). Also affected, but less so, was a strawberry gene with greatest homology to a proton antiporter TT12. These findings suggest two important candidates for anthocyanin transport in strawberry fruit.

This list also confirmed the strawberry genes of the anthocyanin biosynthetic pathway. There appeared to be two $\mathrm{CHS}$ genes 
Table 1 | Differentially expressed genes whose expression profile is MYB10 RNAi/WT > 2 and WT/35S:MYB10>2.

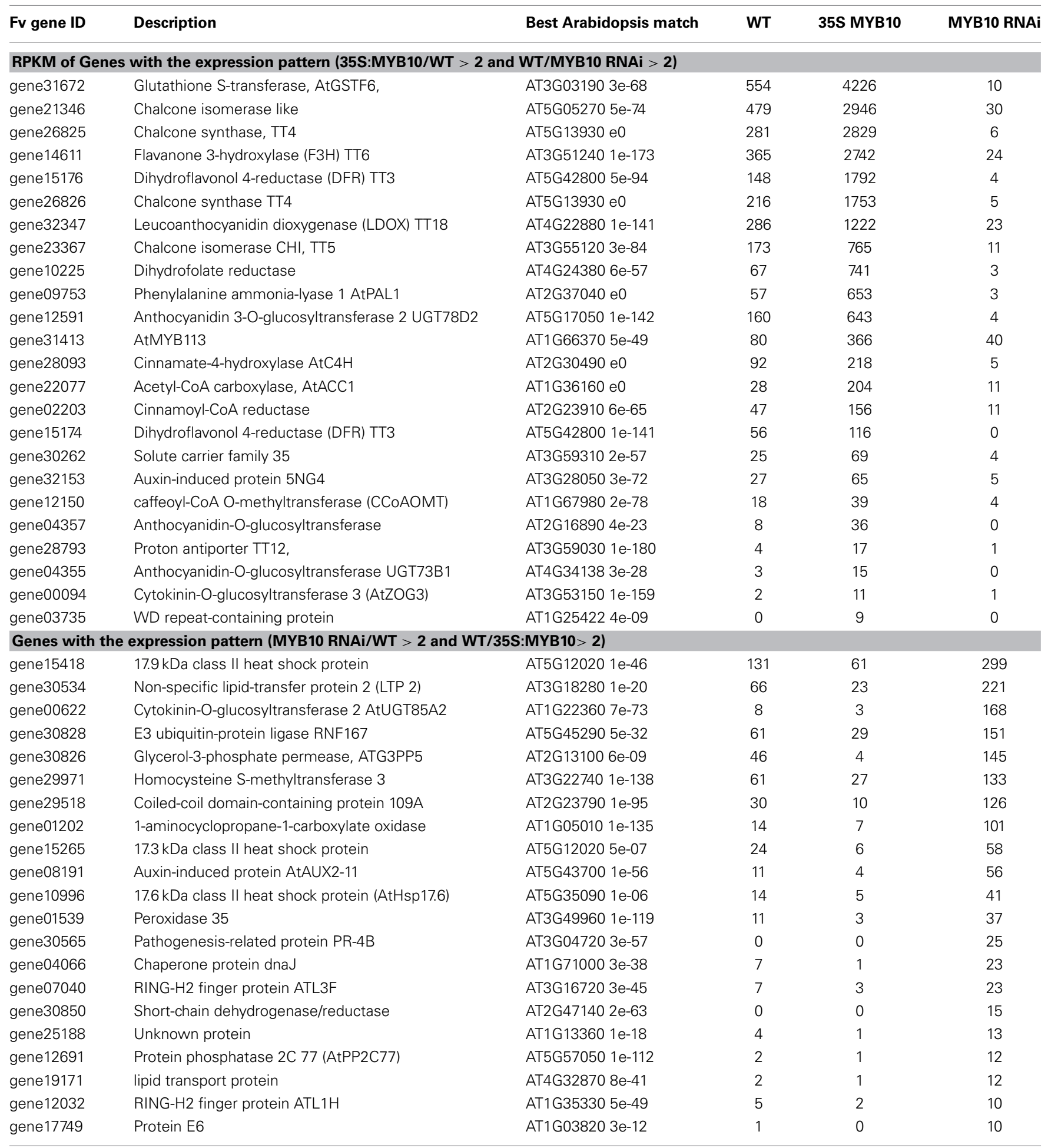

affected which have sequential gene model numbers and appear adjacent to each other in the strawberry genome (Shulaev et al., 2011), suggesting a recent gene duplication. Two $C H I$ genes were differentially expressed; one shares homology to a $C H I$-like gene in Arabidopsis while the other is most similar to the characterized
AtCHI (TT5). Two DFR genes were differentially regulated, while another unknown reductase (gene02203) was annotated in Arabidopsis as a potential cinnamoyl-CoA reductase. The list also provides identification of three potential UFGT genes, with gene12591 being the most likely 3-O-glucosyltransferase as it is 
55\% identical to At5g17050, which specifically glucosylates the 3-position of flavonoids in Arabidopsis.

Of the potential regulators, MYB10 is present in the list (gene31413). However, the WD40 protein identified in this list (gene03735) has very low homology to members of the TTG1 WD40 proteins, so is unlikely to be part of the MBW complex. Four genes unrelated to anthocyanin biosynthesis are on this list, including a dihydrofolate reductase and an acetyl-CoA carboxylase (potentially involved in production of waxes and lipid metabolism).

The opposite comparison was also made; the intersection between the genes that were more than two-fold up-regulated in FvMYB10RNAi-2 (in comparison with wild-type fruit), and were also more than two-fold down-regulated in 35S:FvMYB10 (in comparison with wild-type fruit). The identified genes were all unrelated to anthocyanin metabolism (Table 1). The most affected genes were several small heat shock proteins, as well as several related to stress (pathogen response, peroxidises). An ethylene-related ACO-like gene was up-regulated in white fruit. This suggests that the white fruit may be undergoing a greater stress response during ripening, possibly because of the absence of anthocyanin. Two potential transcription factors were affected: gene08191, which is similar to AtAUX2-11, and the previously named FvAUX/IAA6, and Atl3F, a potential response factor underlying a MAPKinase cascade. In addition, gene12691 is a protein phosphatase highly homologous to Arabidopsis ABA insensitive 2 (AtABI-2), which is negatively regulated by AtMYB20 (Cui et al., 2013).

\section{FVMYB10 IS A KEY ACTIVATOR IN ANTHOCYANIN BIOSYNTHETIC PATHWAY}

It has been previously reported that during fruit development FaMYB10 and FvMYB10 are strongly up-regulated in F. ananassa and F. vesca respectively (Lin-Wang et al., 2010). Similarly, the repressor $F a M Y B 1$ is also up-regulated in $F$. ananassa, albeit to a lesser extent than the activator MYBs, while FvMYB1 shows little change in F. vesca. In the present study, expression analysis was tested using qPCR of mature fruit from four independent 35S:FvMYB10 over-expressing lines, four independent FvMYB10 RNAi lines, two independent FvbHLH33 RNAi lines and four wild-type lines (Figure 5). The expression of FvMYB10 was greatly elevated from 6 to 27-fold in all 35S:FvMYB10 lines and reduced approximately five-fold in two FvMYB10 RNAi lines (FvMYB10 RNAi-2 and FvMYB10 RNAi-4) compared with wild-type controls (Figure 2). The levels of transcripts encoding four anthocyanin biosynthetic genes FvCHS (gene26826-v1.0hybrid), FvF3H (gene14611-v1.0-hybrid), FvDFR (gene15174v1.0-hybrid), FvLDOX (gene32347-v1.0-hybrid), and FvUFGT (gene12591-v1.0-hybrid) were examined among these lines, and showed elevations in all 35S:FvMYB10 lines and reductions in two FvMYB10 RNAi lines (FvMYB10 RNAi-2 and FvMYB10 RNAi4) compared with wild-type controls (Figure 5B). The expression of FvbHLH33 decreased in two FvbHLH33 RNAi lines (Figure 2). However, the reduction of FvbHLH33 transcript did not have a significant impact on the levels of the transcripts of four other transcription factors and the five anthocyanin biosynthetic genes tested. The expression of FvMYB10 was not knocked down in the other two independent FvMYB10 RNAi lines (FvMYB10 RNAi1 and FvMYB10 RNAi-3) and consequently the expression of anthocyanin biosynthetic genes was not affected (Figures 2, 5).

\section{TRANSIENT ASSAY OF STRAWBERRY MYB AND bHLH ACTIVITIES}

Dual luciferase assays were performed in Nicotiana benthamiana to examine FvMYB10 and FvMYB1 activity against the Arabidopsis DFR (At5g42800), F. vesca DFR and UFGT promoters and also to investigate interactions between FvMYBs and FvbHLHs. As shown in Figure 6A, FvMYB10 can activate the Arabidopsis DFR, F. vesca DFR (gene15174-v1.0-hybrid) and UFGT (gene12591-v1.0-hybrid) promoters, but only in the presence of FvbHLH33. This activation was strongly reduced when the repressor FvMYB1 was co-transformed with FvMYB10 and FvbHLH33.

In apple it has been shown that a 23-bp sequence motif in the MdMYB10 promoter region is a target of the apple MdMYB10 protein itself and the number of repeats in this motif correlates with an increase in trans-activation by the MdMYB10 protein (Espley et al., 2009). This motif was not found in the promoter region of $F v M Y B 10$. However, the activity of the FvMYB10 promoter was slightly induced by both FvMYB10 and FvMYB1 protein (Figure 6A). An analysis of cis-acting regulatory motifs within the FvMYB10 promoter region showed a number of MYB-related elements (http://www.dna.affrc.go.jp/PLACE/) which could account for this, as well as bHLH-related E-boxes (Supplementary Table 7). There was a less significant induction observed in either the FvMYB1 or FvbHLH33 promoters when co-transformed with the transcription factors tested in the assay.

The activity of the AtDFR promoter was reduced when either FvMYB10 RNAi or FvbHLH33 RNAi was co-transformed with FvMYB10 and FvbHLH33 (Figure 6B). The AtDFR promoter was used in the transient RNAi assay because its activity was much stronger than the F.vesca DFR and UFGT promoters when induced by FvMYB10 and FvbHLH33 proteins.

\section{EXPRESSION OF FvMYB10 AFFECTS OTHER MEMBERS OF THE R2R3 MYB FAMILY}

The MYB family of transcription factors has been reported to interact with each other in various networks. To ascertain if MYB10 co-regulates other MYBs, analysis of RNA-seq counts in the WT verses transgenic lines was made. Clustal analysis of MYBs with altered RPKM values are shown in Figure 7. A total of 12 MYBs, which showed values greater than 20 RPKM in at least one of the libraries, were affected by the over-expression of MYB10, or its knockdown, or knockdown of bHLH33 (shown in red boxes, Figure 7).

Of interest was FvMYB89, an R2R3 MYB which was upregulated by the knock-down of MYB10. Expression of FvMYB89 was not detected in the WT at harvest, but increased to RPKM values of 20-50 when MYB10 was knocked down. FvMYB89 is homologous to AtMYB21, a gene normally expressed only in floral development, promoting petal and stamen growth (Reeves et al., 2012). The homolog of MYB89 is also affected in F. ananassa fruit when MYB10 is transiently knocked-down, where it was named $F a E O B I I$ because of its sequence similarity with $E O B I I$ 


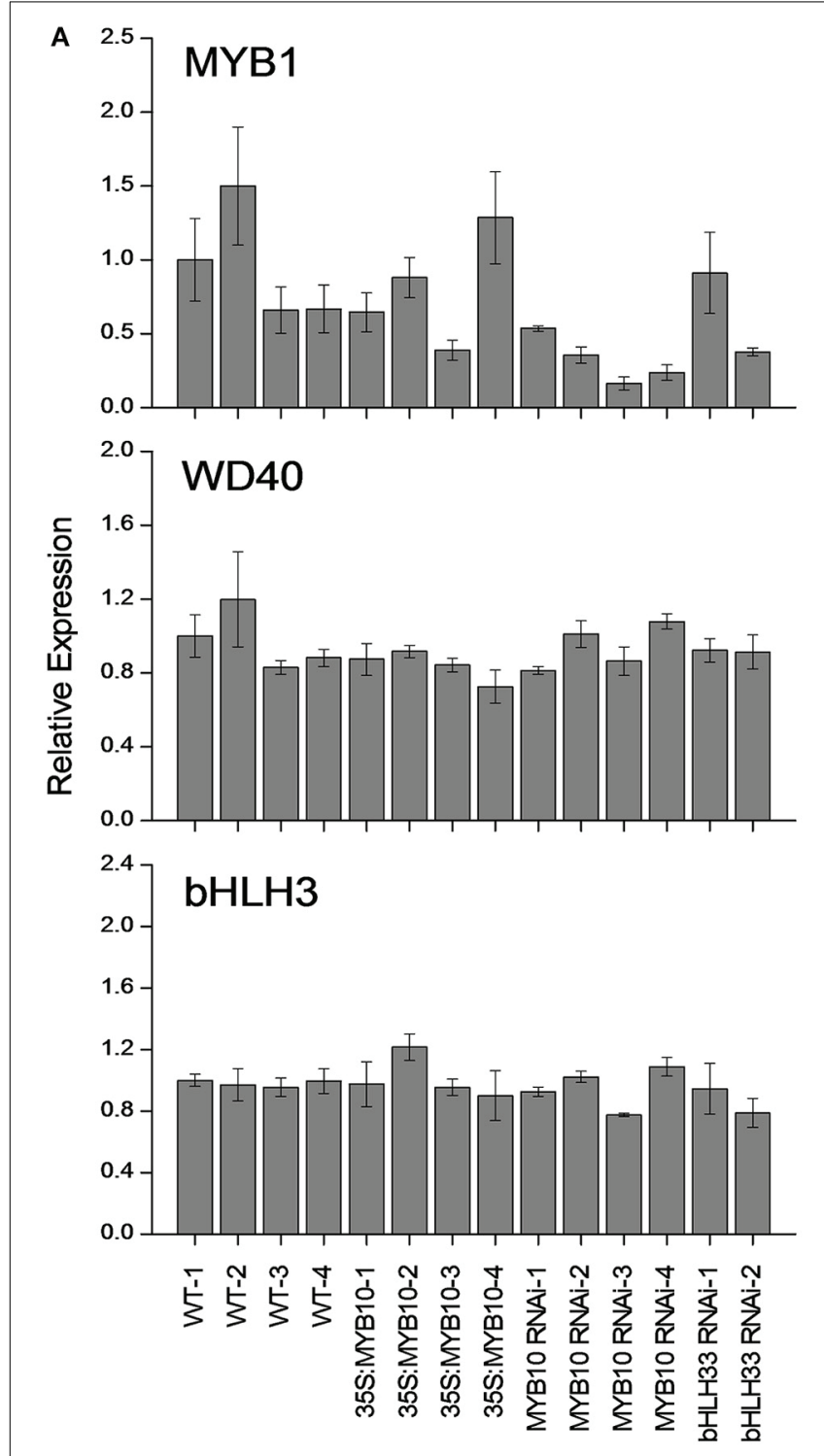

FIGURE 5 | Expression analysis of strawberry anthocyanin-related transcription factors and biosynthetic genes. GPCR analysis of mature F. vesca fruit from four wild-type lines, four 35S: FVMYB10 lines, four FVMYB10 RNAi lines and two FvbHLH33 RNAi lines. (A) MYB1, putative repressor; bHLH3, potential partner; WD40, AtTTG1 like. (B) $C H S$, chalcone synthase; $F 3 H$, flavanone $3 \beta$-hydroxylase; $D F R$, dihydroflavonol 4-reductase;

\section{B}
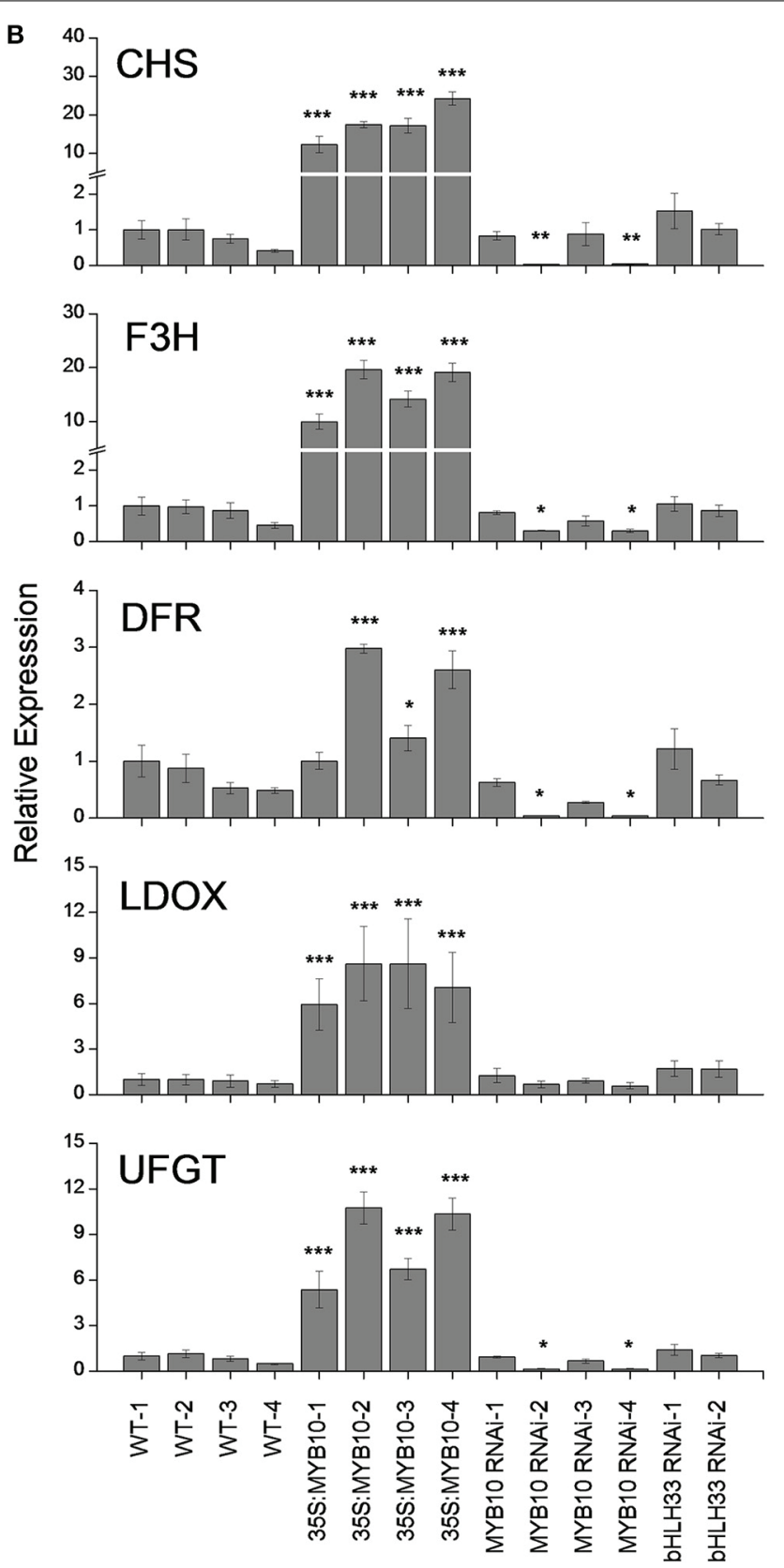

LDOX, leucoanthocyanidin dioxygenase; UFGT, uridine diphosphate (UDP)-glucose:flavonoid 3-O-glycosyltransferase. Transcript abundance was calculated relative to the geometric mean of GAPDH, PP2a and UBC9. Errors bars are the $S E$ for three biological replicates. Statistical significance between transgenic lines and wild-type controls was determined by One-Way ANOVA: ${ }^{*} P<0.05 ;{ }^{*} P<0.01 ;{ }^{* *} P<0.001$.
(Emission of Benzenoids II) of Petunia (Medina-Puche et al., 2014). This TF has been previously characterized as a regulator of volatile phenylpropanoids in petunia flowers, such as phenylethyl alcohol, benzylbenzoate, eugenol, and isoeugenol (Spitzer-Rimon et al., 2010). FvMYB1, the repressor of anthocyanin biosynthesis showed a pattern similar to qPCR analysis (Figure 5), down slightly in the MYB10 RNAi lines. FvMYB17 is another candidate repressor, and is well expressed, and is up-regulated in $35 \mathrm{~S}$ MYB10 lines, and down-regulated in knock out lines of MYB10.

\section{ANALYSIS OF FRUIT VOLATILES}

Because of the observation that several floral-related MYBs were affected by the presence or absence of MYB10, an analysis of fruit volatiles was performed using GC-MS (7890A-5975C, Agilent 


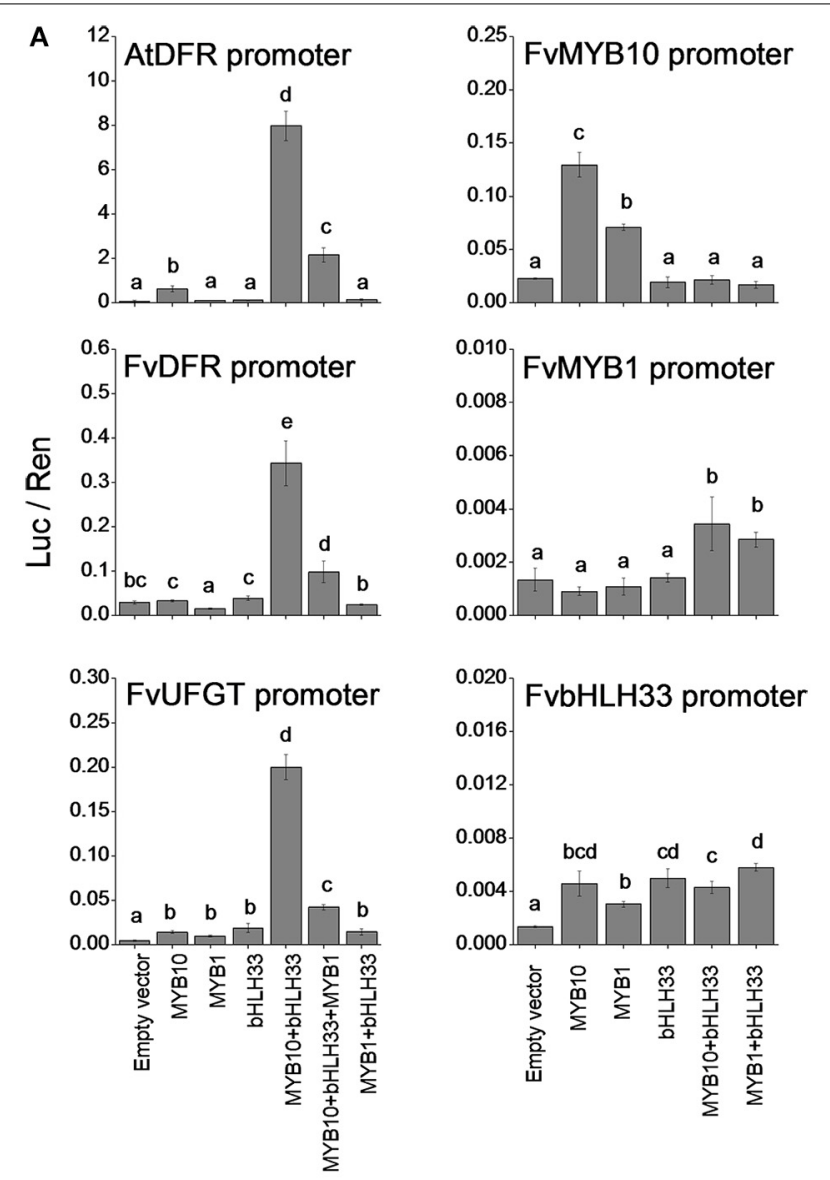

B

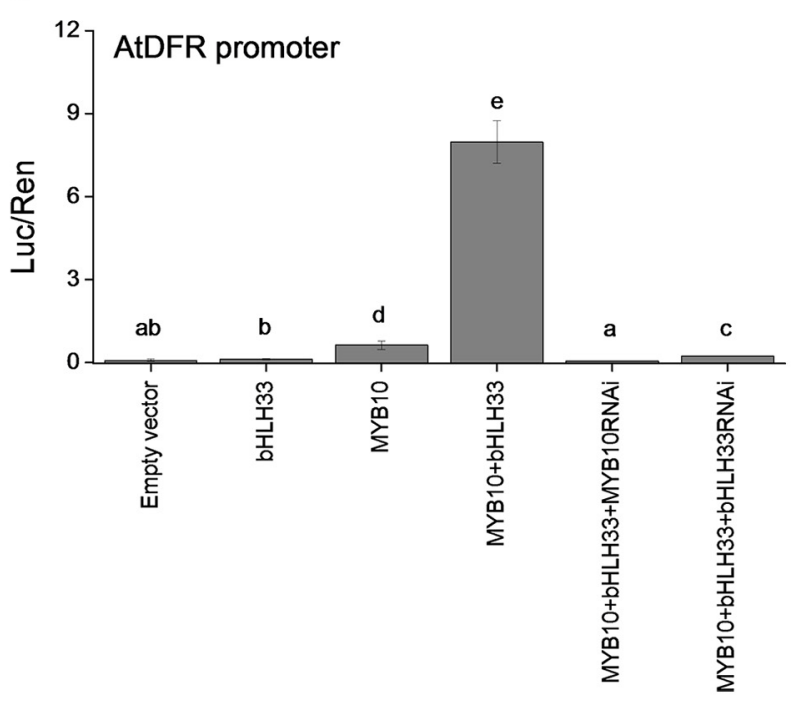

FIGURE 6 | Transient assay of the interaction between transcription factors and promoters of strawberry anthocyanin-related steps. The dual luciferase assay, in Nicotiana benthamiana shows promoter activity expressed as a ratio of candidate promoter luciferase (Luc) to 35 S Renilla (Ren). (A) FvMYB10 with FvbHLH33 activates the promoters of AtDFR, FVDFR, and FVUFGT, and FvMYB1 prevented the activation of these three promoters. The FVMYB10 promoter was slightly induced by FVMYB10 and

(Continued)

\section{FIGURE 6 | Continued}

FvMYB1. No significant induction was observed in either the FvMYB1 or FvbHLH33 promoters when co-transformed with the transcription factors tested in the assay. (B) The activity of the AtDFR promoter was significantly reduced when either FvMYB10 RNAi or FvbHLH33 RNAi was co-transformed with FvMYB10 and FvbHLH33. Error bars are the SE for four replicate reactions. Statistical significance was determined by One-Way ANOVA; significant differences between means (LSD, $P<0.05$ ) are indicated where letters $(a, b, c$, etc.) above the bar differ.

Technologies). Esters have been reported as some of the most important sensory qualities for strawberry (Azodanlou et al., 2003). In our study, some significant differences were observed, particularly when comparing the over-expressing lines with the WT (Figure 8). The acetic acids, butyl ester and hexyl ester and butanoic acid, ethyl ester were all at higher concentrations, and acetic acid, and octyl ester were at lower concentrations in 35S:FvMYB10 lines. In contrast, the silenced lines showed little change from the WT, except for an increase in 2-heptanone. Whilst this compound has been identified in the white strawberry, Fragaria chiloensis, it is not necessarily a defining aroma volatile for white berries (Prat et al., 2014).

\section{DISCUSSION}

In the present study, a range of transgenic lines was used to test the specific roles of anthocyanin biosynthesis regulators in woodland strawberry. The use of independent over-expression and knockdown lines together with both targeted and global expression analysis provided strong evidence for these roles as well as suggesting the possibility of other seemingly unrelated effects such as volatile production and stress response.

The phenotypic evidence suggest no redundancy for MYB10; over-expressing lines had greatly increased anthocyanin concentrations whilst the two knock-down lines with the least MYB10 expression had undetectable concentrations of both the major pigment contributing compounds, cyanidin glucoside, and pelargonidin glucoside. In contrast, knock-down of a potential partner of MYB10, bHLH33, had no phenotypic effect, suggesting a level of redundancy of the bHLH factors. It is clear that in a heterologous system, such as the promoter interaction assays presented here, bHLH33 is essential for regulation of anthocyanin biosynthesis steps. This does not appear to be the case in stably transformed strawberry where the lack of $b H L H 33$ expression does not affect anthocyanin concentrations.

The over-expression of MYB10 did not alter other flavonoid concentrations in the fruit. Of all the metabolites tested, only pcoumaryl glucose was affected. This observation differs from that in apple, for example, where the over-expression of the homologous apple MYB resulted in increased flavon-3-ols and flavonols (Espley et al., 2013). This suggests a defined role for MYB10 in strawberry, limited to the anthocyanin branch of flavonoid production. Targeted gene expression analysis confirmed the function of MYB10 as the regulator of anthocyanin-related enzymes throughout the pathway from the first committed step, CHS, to the final glycosylation step, UFGT. In $F$. ananassa it has been reported that LDOX (also termed ANS) is not regulated by FaMYB10 (Medina-Puche et al., 2014). In FaMYB10 silenced lines 


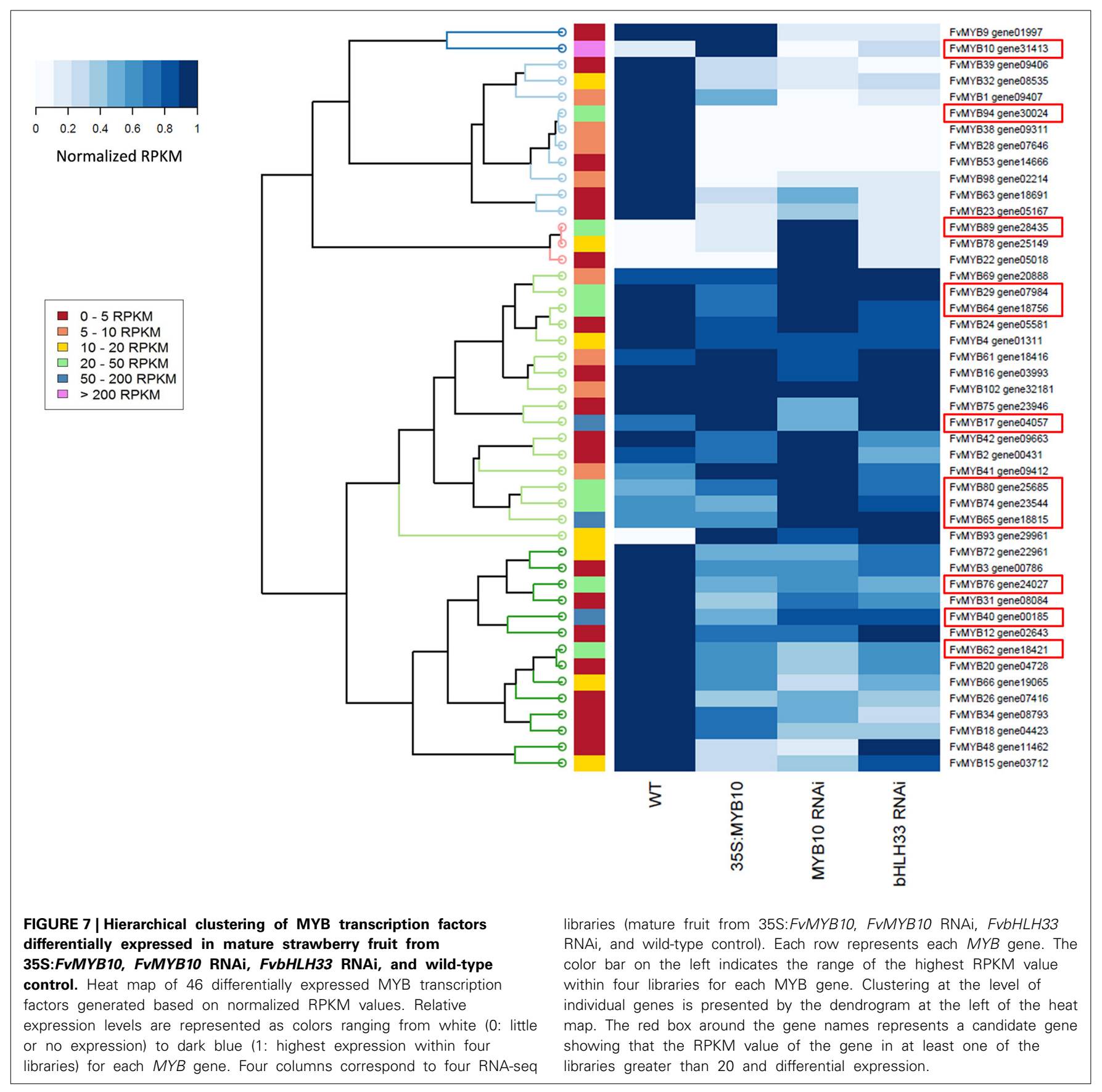

the transcript abundance was unchanged for $L D O X$, unlike for other genes in the anthocyanin pathway. The results here for F. vesca showed an increase in transcription of $L D O X$ in MYB10 over-expressing lines suggesting a role for MYB10 in LDOX transcription as with all the other anthocyanin biosynthesis genes tested. In FvMYB10 silenced lines, LDOX shows a similar transcript level to the WT and is not statistically different, although the heavily silenced lines (lines 2 and 4 ) do have the least expression, concomitant with CHS, F3H, DFR, and UFGT. It appears then that FaMYB10 and FvMYB10 may have different roles in regulating $L D O X$ transcription.
Data generated by RNA seq analysis confirmed MYB10 regulation of the anthocyanin pathway, and also revealed a more detailed picture of possible gene family members. These included possible duplications of CHS, CHI, DFR, and UFGT which all showed some level of transcription. Interestingly, the most differentially expressed gene was shown to be a glutathione Stransferase, homologous to a known anthocyanin transporter in Arabidopsis. In the list of genes both down-regulated in MYB10 over-expression lines, and up-regulated in white knockdown lines, there were several stress-related genes. This suggested that an absence of anthocyanins during fruit maturation 


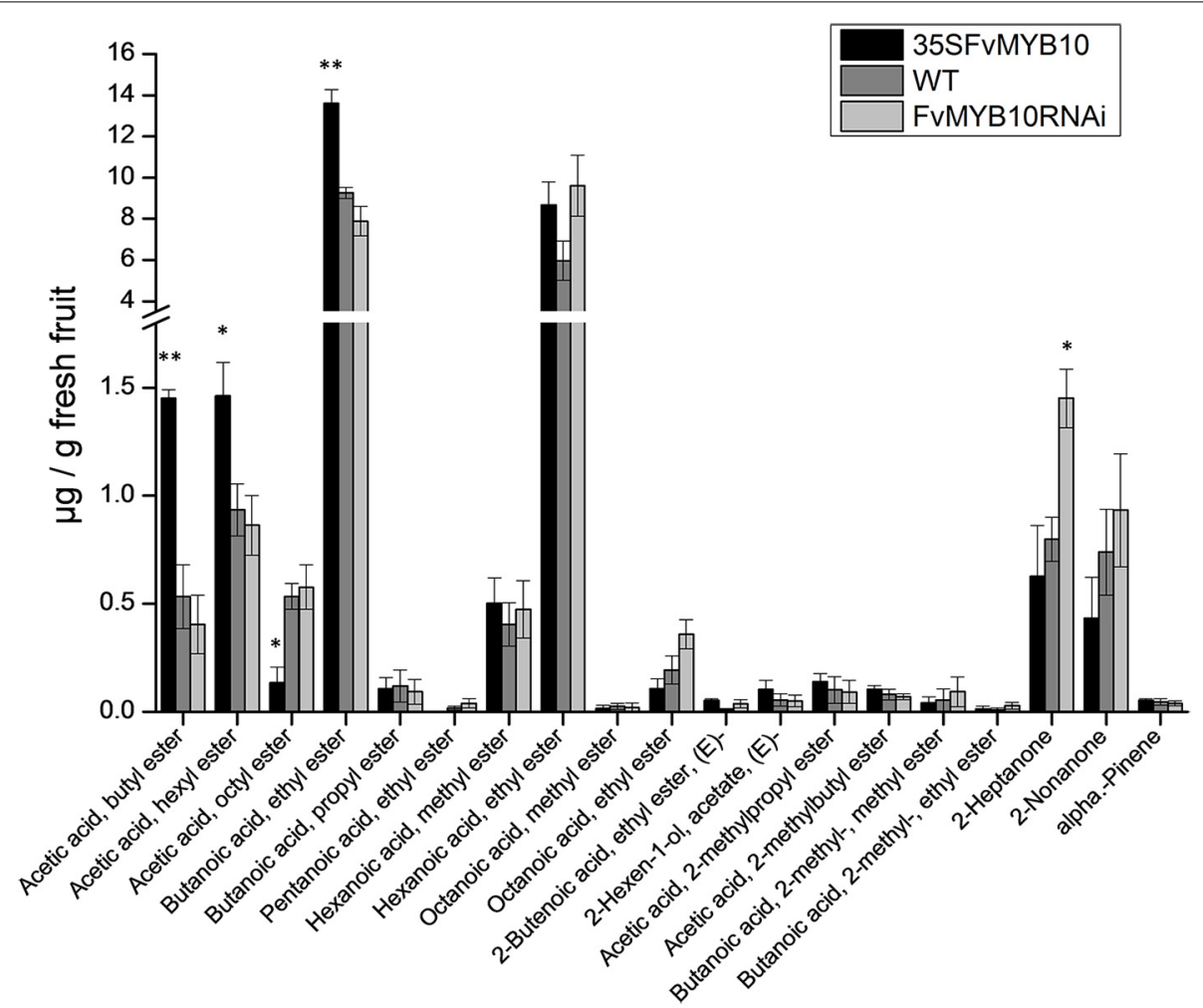

FIGURE 8 | Fruit volatiles of mature strawberry fruit from 35S:FvMYB10, FvMYB10 RNAi, and wild-type plants. GC-MS quantification of fruit volatiles. The acetic acids, butyl ester and hexyl ester and butanoic acid, ethyl ester were significantly higher, and acetic acid and octyl ester were significantly lower in 35S: FvMYB10 than in the wild-type control and FVMYB10 RNAi. FVMYB10 RNAi fruit had the highest concentration of 2-heptanone. Statistical significance between transgenic lines and wild-type controls was determined by One-Way ANOVA: ${ }^{*} P<0.05 ;{ }^{* *} P<0.01$. leads to increase in cellular stress. Recently, increasing ascorbate in strawberries by transformation of the key regulatory gene GDP-galactose phosphorylase resulted in increased polyphenolics (Bulley et al., 2012). Ascorbate is a key compound in stress responses in plants supporting the idea that there may be a direct link between stress and polyphenolic concentrations.

Functional analysis of MYB10 using transient transformation of tobacco also showed that the TF was able to interact with and elevate the activity of key genes in the anthocyanin pathway, DFR and UFGT. However, this activation only occurred with the co-transformation of bHLH33. When the repressor TF MYB1 was also included with MYB10 and bHLH33 this activation was repressed. Transient transformation of bHLH33 on its own did not produce promoter activation. To test for auto-activation, MYB10, MYB1, and bHLH33 promoters were all tested against their proteins. Autoregulation of anthocyanin-related MYBs has been previously demonstrated but this was due to a novel motif not present in the FvMYB10 promoter (Espley et al., 2009). The results shown here suggest that the FvMYB10 promoter can be activated by MYB10 protein despite the apparent lack of autoregulatory motifs found in the sequence. There are, however, a number of general MYB-related motifs within the isolated strawberry promoter region which may explain activation. It was interesting to note that transactivation was higher in the presence of MYB10 than of MYB1. The activation of FvMYB10 with MYB1 suggests possible functionality of other anthocyaninrelated MYBs. Interestingly, the MYB1 promoter, which also contains predicted MYB-related cis-acting elements, was apparently unresponsive to either the MYB10 or MYB1 proteins although there was some evidence for activation when co-infiltrated with bHLH33. Co-infiltration with bHLH33 produced no activation for the FvMYB10 promoter, in contrast to similar experiments using apple MYB10 (Espley et al., 2007) where a combination of MYB and BHLH proteins had a stronger activation effect on the MYB10 promoter than MYB10 alone.

This work adds to the existing knowledge of anthocyanin regulation in strawberry and confirms that both cultivated and wild strawberry share similar anthocyanin regulation (Lin-Wang et al., 2010; Medina-Puche et al., 2014). With silencing of MYB10, there was some difference in the anthocyanin metabolites altered but this is likely to be due to the original anthocyanin composition in fruit from the two species. In both FvMYB10 and FaMYB10 transcription was shown to be fruit specific and correlated with anthocyanin accumulation throughout fruit ripening. Further, both MYB10s also appear to exert regulation on a broad range of genes involved in the anthocyanin pathway. However, silencing of FaMYB10 had little or no effect on $L D O X$ (ANS), which also appears to be the case for $F v M Y B 10$, where there was no statistical difference, in contrast to the other genes tested (CHS, $F 3 H, D F R$, and UFGT). Conversely, stable over-expression of 
FvMYB10 did lead to an increase in $L D O X$ transcript levels suggesting that FvMYB10 may have some regulating function, unless the increased transcript level is the result of increased pathway flux.

The transcriptome data showed that other MYBs, even those not associated with anthocyanin accumulation, were affected by over-expression of MYB10. Several of these MYBs are implicated in regulating volatile concentrations. In MYB10 silenced F.ananassa, the expression of a volatile-related MYB, FaEOBII (GENE28435), was shown to be down-regulated (Medina-Puche et al., 2014). In F. Vesca the expression of this gene was higher in MYB10 knocked down fruit compared with WT or FvMYB10 over-expressing lines (Figure 7). MYB TF regulation was also suggested for a $\mathrm{F}$. Ananassa $\mathrm{O}$-methyltransferase gene $(\mathrm{FaOMT})$ involved in mesifurane production (Zorrilla-Fontanesi et al., 2012). The functional allele, as opposed to the non-functional allele, of $\mathrm{FaOMT}$ contains both MYB and bHLH-related binding domains in the promoter sequence which may account for the relatively high expression and consequent mesifurane content. Whilst this may not be relevant for $F$. Vesca, which has a relatively low level of FvOMT expression and low mesifurane concentration (Zorrilla-Fontanesi et al., 2012), it does further suggest the possibility for some role for MYBs in the regulation of strawberry volatile production. Further work is required to determine what, if any, direct effect the FvMYB10 protein may play on strawberry volatile production.

In summary, our study shows that both real-time quantitative PCR and transcriptomic analysis showed a strong correlation between the expression of FvMYB10 and the concentrations of anthocyanin in 35S:FvMYB10 lines, FvMYB10 RNAi lines and wild-type controls. In contrast, $F v b H L H 33$, which is a potential $b H L H$ partner for $F v M Y B 10$, did not affect the anthocyanin pathway when knocked down using an RNAi construct. The results suggested the possible redundancy of $b H L H$ partners in strawberry. In transient transactivation assays, FvMYB10, co-expressed with FvbHLH33, strongly activated the AtDFR, $F v D F R$, and FvUFGT promoters, while knocking down either FvMYB10 or FvbHLH33 significantly reduced the activity of the AtDFR promoter in Nicotiana benthamiana plants. FvMYB1, an R2R3 repressor MYB, proved to be a negative regulator of the strawberry anthocyanin biosynthetic pathway in transient assays.

There is a strong relationship with MYB10 expression and other fruit ripening processes and it has also been demonstrated that FaMYB10 expression and consequent anthocyanin concentration is effected by hormone levels in the fruit (Medina-Puche et al., 2014). It is likely that hormonal balance would similarly influence FvMYB10. The next challenge is to further elucidate the specific genes and hormonal cues that determine MYB10 expression in strawberry.

\section{ACKNOWLEDGMENTS}

We thank William Laing for comments on the manuscript, Peter McAtee for assistance with heat map methods, Luca Ding for assistance with RNA preparation, Tim Holmes for photography and Monica Dragulescu for looking after plants in the glasshouse.

\section{SUPPLEMENTARY MATERIAL}

The Supplementary Material for this article can be found online at: http://www.frontiersin.org/journal/10.3389/fpls.2014. 00651/abstract

\section{REFERENCES}

Aharoni, A., De Vos, C. H., Wein, M., Sun, Z., Greco, R., Kroon, A., et al. (2001). The strawberry FaMYB1 transcription factor suppresses anthocyanin and flavonol accumulation in transgenic tobacco. Plant J. 28, 319-332. doi: 10.1046/j.1365-313X.2001.01154.x

Allan, A. C., Hellens, R. P., and Laing, W. A. (2008). MYB transcription factors that colour our fruit. Trends Plant Sci. 13, 99-102. doi: 10.1016/j.tplants.2007.11.012

Azodanlou, R., Darbellay, C., Luisier, J. L., Villettaz, J. C., and Amado, R. (2003). Quality assessment of strawberries (Fragaria species). J. Agric. Food Chem. 51, 715-721. doi: 10.1021/jf0200467

Baudry, A., Caboche, M., and Lepiniec, L. (2006). TT8 controls its own expression in a feedback regulation involving TTG1 and homologous MYB and bHLH factors, allowing a strong and cell-specific accumulation of flavonoids in Arabidopsis thaliana. Plant J. 46, 768-779. doi: 10.1111/j.1365313X.2006.02733.x

Blankenberg, D., Gordon, A., Von Kuster, G., Coraor, N., Taylor, J., Nekrutenko, A., et al. (2010). Manipulation of FASTQ data with galaxy. Bioinformatics 26, 1783-1785. doi: 10.1093/bioinformatics/btq281

Bulley, S., Wright, M., Rommens, C., Yan, H., Rassam, M., Lin-Wang, K., et al. (2012). Enhancing ascorbate in fruits and tubers through over-expression of the l-galactose pathway gene GDP-l-galactose phosphorylase. Plant Biotechnol. J. 10, 390-397. doi: 10.1111/j.1467-7652.2011.00668.x

Butelli, E., Titta, L., Giorgio, M., Mock, H. P., Matros, A., Peterek, S., et al. (2008). Enrichment of tomato fruit with health-promoting anthocyanins by expression of select transcription factors. Nat. Biotechnol. 26, 1301-1308. doi: 10.1038/nbt.1506

Chang, S., Puryear, J., and Cairney, J. (1993). A simple and efficient method for isolating RNA from pine trees. Plant Mol. Biol. Rep. 11, 113-116. doi: 10.1007/BF02670468

Cui, M. H., Yoo, K. S., Hyoung, S., Nguyen, H. T., Kim, Y. Y., Kim, H. J., et al. (2013). An Arabidopsis R2R3-MYB transcription factor, AtMYB20, negatively regulates type $2 \mathrm{C}$ serine/threonine protein phosphatases to enhance salt tolerance. FEBS Lett. 587, 1773-1778. doi: 10.1016/j.febslet.2013.04.028

Davies, K. M., and Espley, R. V. (2013). Opportunities and challenges for metabolic engineering of secondary metabolite pathways for improved human health characters in fruit and vegetable crops. N.Z. J. Crop Hortic. Sci. 41, 154-177. doi: 10.1080/01140671.2013.793730

Dubos, C., Le Gourrierec, J., Baudry, A., Huep, G., Lanet, E., Debeaujon, I., et al. (2008). MYBL2 is a new regulator of flavonoid biosynthesis in Arabidopsis thaliana. Plant J. 55, 940-953. doi: 10.1111/j.1365-313X.2008.03564.x

Espley, R. V., Bovy, A., Bava, C., Jaeger, S. R., Tomes, S., Norling, C., et al. (2013). Analysis of genetically modified red-fleshed apples reveals effects on growth and consumer attributes. Plant Biotechnol. J. 11, 408-419. doi: 10.1111/pbi.12017

Espley, R. V., Brendolise, C., Chagne, D., Kutty-Amma, S., Green, S., Volz, R., et al. (2009). Multiple repeats of a promoter segment causes transcription factor autoregulation in red apples. Plant Cell 21, 168-183. doi: 10.1105/tpc.108.059329

Espley, R. V., Butts, C. A., Laing, W. A., Martell, S., Smith, H., McGhie, T. K., et al. (2014). Dietary flavonoids from modified apple reduce inflammation markers and modulate gut microbiota in mice. J. Nutr. 144, 146-154. doi: 10.3945/jn.113.182659

Espley, R. V., Hellens, R. P., Putterill, J., Stevenson, D. E., Kutty-Amma, S., and Allan, A. C. (2007). Red colouration in apple fruit is due to the activity of the MYB transcription factor, MdMYB10. Plant J. 49, 414-427. doi: 10.1111/j.1365313X.2006.02964.x

Fait, A., Hanhineva, K., Beleggia, R., Dai, N., Rogachev, I., Nikiforova, V. J., et al. (2008). Reconfiguration of the achene and receptacle metabolic networks during strawberry fruit development. Plant Physiol. 148, 730-750. doi: 10.1104/pp.108.120691

Fischer, T. C., Mirbeth, B., Rentsch, J., Sutter, C., Ring, L., Flachowsky, H., et al. (2014). Premature and ectopic anthocyanin formation by silencing of anthocyanidin reductase in strawberry (Fragaria $\times$ ananassa). New Phytol. 201, 440-451. doi: 10.1111/nph.12528 
Folta, K. M., and Davis, T. M. (2006). Strawberry Genes and Genomics. Crit. Rev. Plant Sci. 25, 399-415. doi: 10.1080/07352680600824831

Folta, K. M., and Dhingra, A. (2006). Invited review: transformation of strawberry: the basis for translational genomics in Rosaceae. In Vitro Cell. Dev. Biol. Plant 42, 482-490. doi: 10.1079/IVP2006807

Gleave, A. P. (1992). A versatile binary vector system with a T-DNA organizationalstructure conducive to efficient integration of cloned DNA into the plant genome. Plant Mol. Biol. 20, 1203-1207. doi: 10.1007/BF00028910

Gonzalez, A., Zhao, M., Leavitt, J. M., and Lloyd, A. M. (2008). Regulation of the anthocyanin biosynthetic pathway by the TTG1/bHLH/Myb transcriptional complex in Arabidopsis seedlings. Plant J. 53, 814-827. doi: 10.1111/j.1365313X.2007.03373.x

Griesser, M., Hoffmann, T., Bellido, M. L., Rosati, C., Fink, B., Kurtzer, R., et al. (2008). Redirection of flavonoid biosynthesis through the down-regulation of an anthocyanidin glucosyltransferase in ripening strawberry fruit. Plant Physiol. 146, 1528-1539. doi: 10.1104/pp.107.114280

Hannum, S. M. (2004). Potential impact of strawberries on human health: a review of the science. Crit. Rev. Food Sci. Nutr. 44, 1-17. doi: $10.1080 / 10408690490263756$

Hellens, R. P., Allan, A. C., Friel, E. N., Bolitho, K., Grafton, K., Templeton, M. D., et al. (2005). Transient plant expression vectors for functional genomics, quantification of promoter activity and RNA silencing. Plant Methods 1:13. doi: 10.1186/1746-4811-1-13

Jaakola, L. (2013). New insights into the regulation of anthocyanin biosynthesis in fruits. Trends Plant Sci. 18, 477-483. doi: 10.1016/j.tplants.2013.06.003

Jin, H., Cominelli, E., Bailey, P., Parr, A., Mehrtens, F., Jones, J., et al. (2000). Transcriptional repression by AtMYB4 controls production of UV-protecting sunscreens in Arabidopsis. EMBO J. 19, 6150-6161. doi: 10.1093/emboj/19.22.6150

Li, H., and Durbin, R. (2009). Fast and accurate short read alignment with Burrows-Wheeler transform. Bioinformatics 25, 1754-1760. doi: 10.1093/bioinformatics/btp324

Lin-Wang, K., Bolitho, K., Grafton, K., Kortstee, A., Karunairetnam, S., McGhie, T. K., et al. (2010). An R2R3 MYB transcription factor associated with regulation of the anthocyanin biosynthetic pathway in Rosaceae. BMC Plant Biol. 10:50. doi: 10.1186/1471-2229-10-50

Lin-Wang, K., Micheletti, D., Palmer, J., Volz, R., Lozano, L., Espley, R., et al. (2011). High temperature reduces apple fruit colour via modulation of the anthocyanin regulatory complex. Plant Cell Environ. 34, 1176-1190. doi: 10.1111/j.13653040.2011.02316.x

Medina-Puche, L., Cumplido-Laso, G., Amil-Ruiz, F., Hoffmann, T., Ring, L., Rodriguez-Franco, A., et al. (2014). MYB10 plays a major role in the regulation of flavonoid/phenylpropanoid metabolism during ripening of Fragaria ananassa fruits. J. Exp. Bot. 65, 401-417. doi: 10.1093/jxb/ert377

Oosumi, T., Gruszewski, H. A., Blischak, L. A., Baxter, A. J., Wadl, P. A., Shuman, J. L., et al. (2006). High-efficiency transformation of the diploid strawberry (Fragaria vesca) for functional genomics. Planta 223, 1219-1230. doi: 10.1007/s00425-005-0170-3

Pfaffl, M. W. (2001). A new mathematical model for relative quantification in realtime RT-PCR. Nucleic Acids Res. 29, e45. doi: 10.1093/nar/29.9.e45

Prat, L., Espinoza, M. I., Agosin, E., and Silva, H. (2014). Identification of volatile compounds associated with the aroma of white strawberries (Fragaria chiloensis). J. Sci. Food Agric. 94, 752-759. doi: 10.1002/jsfa.6412

Reeves, P. H., Ellis, C. M., Ploense, S. E., Wu, M. F., Yadav, V., Tholl, D., et al. (2012). A regulatory network for coordinated flower maturation. PLoS Genet. 8:e1002506. doi: 10.1371/journal.pgen.1002506

Rowan, D. D., Cao, M., Lin-Wang, K., Cooney, J. M., Jensen, D. J., Austin, P. T., et al. (2009). Environmental regulation of leaf colour in red 35S:PAP1 Arabidopsis thaliana. New Phytol. 182, 102-115. doi: 10.1111/j.1469-8137.2008.02737.x

Salvatierra, A., Pimentel, P., Moya-Leon, M. A., and Herrera, R. (2013). Increased accumulation of anthocyanins in Fragaria chiloensis fruits by transient suppression of FcMYB1 gene. Phytochemistry 90, 25-36. doi: 10.1016/j.phytochem.2013.02.016
Schaart, J. G., Dubos, C., Romero De La Fuente, I., Van Houwelingen, A. M., De Vos, R. C., Jonker, H. H., et al. (2013). Identification and characterization of MYB-bHLH-WD40 regulatory complexes controlling proanthocyanidin biosynthesis in strawberry (Fragaria x ananassa) fruits. New Phytol. 197, 454-467. doi: 10.1111/nph.12017

Shulaev, V., Sargent, D. J., Crowhurst, R. N., Mockler, T. C., Folkerts, O., Delcher, A. L., et al. (2011). The genome of woodland strawberry (Fragaria vesca). Nat. Genet. 43, 109-116. doi: 10.1038/ng.740

Snowden, K. C., Simkin, A. J., Janssen, B. J., Templeton, K. R., Loucas, H. M., Simons, J. L., et al. (2005). The Decreased apical dominancel/Petunia hybrida CAROTENOID CLEAVAGE DIOXYGENASE8 gene affects branch production and plays a role in leaf senescence, root growth, and flower development. Plant Cell 17, 746-759. doi: 10.1105/tpc.104.027714

Spitzer-Rimon, B., Marhevka, E., Barkai, O., Marton, I., Edelbaum, O., Masci, T., et al. (2010). EOBII, a gene encoding a flower-specific regulator of phenylpropanoid volatiles' biosynthesis in Petunia. Plant Cell 22, 1961-1976. doi: $10.1105 /$ tpc. 109.067280

Sun, Y., Li, H., and Huang, J. R. (2012). Arabidopsis TT19 functions as a carrier to transport anthocyanin from the cytosol to tonoplasts. Mol Plant 5, 387-400. doi: $10.1093 / \mathrm{mp} / \mathrm{ssr} 110$

Tamura, K., Peterson, D., Peterson, N., Stecher, G., Nei, M., and Kumar, S. (2011). MEGA5: molecular evolutionary genetics analysis using maximum likelihood, evolutionary distance, and maximum parsimony methods. Mol. Biol. Evol. 28, 2731-2739. doi: 10.1093/molbev/msr121

Toufektsian, M. C., De Lorgeril, M., Nagy, N., Salen, P., Donati, M. B., Giordano, L., et al. (2008). Chronic dietary intake of plant-derived anthocyanins protects the rat heart against ischemia-reperfusion injury. J. Nutr. 138, $747-752$.

Traka, M. H., and Mithen, R. F. (2011). Plant science and human nutrition: challenges in assessing health-promoting properties of phytochemicals. Plant Cell 23, 2483-2497. doi: 10.1105/tpc.111.087916

Trapnell, C., Williams, B. A., Pertea, G., Mortazavi, A., Kwan, G., Van Baren, M. J., et al. (2010). Transcript assembly and quantification by RNA-Seq reveals unannotated transcripts and isoform switching during cell differentiation. Nat. Biotechnol. 28, 511-515. doi: 10.1038/nbt.1621

Wang, M. Y., Macrae, E., Wohlers, M., and Marsh, K. (2011). Changes in volatile production and sensory quality of kiwifruit during fruit maturation in Actinidia deliciosa 'Hayward' and A. chinensis 'Hort16A'. Postharvest Biol. Technol. 59, 16-24. doi: 10.1016/j.postharvbio.2010.08.010

Zorrilla-Fontanesi, Y., Rambla, J. L., Cabeza, A., Medina, J. J., Sanchez-Sevilla, J. F., Valpuesta, V., et al. (2012). Genetic analysis of strawberry fruit aroma and identification of O-Methyltransferase FaOMT as the locus controlling natural variation in mesifurane content. Plant Physiol. 159, 851-870. doi: $10.1104 /$ pp.111.188318

Conflict of Interest Statement: The authors declare that the research was conducted in the absence of any commercial or financial relationships that could be construed as a potential conflict of interest.

Received: 17 July 2014; accepted: 03 November 2014; published online: 19 November 2014.

Citation: Lin-Wang K, McGhie TK, Wang M, Liu Y, Warren B, Storey R, Espley RV and Allan AC (2014) Engineering the anthocyanin regulatory complex of strawberry (Fragaria vesca). Front. Plant Sci. 5:651. doi: 10.3389/fpls.2014.00651

This article was submitted to Plant Metabolism and Chemodiversity, a section of the journal Frontiers in Plant Science.

Copyright () 2014 Lin-Wang, McGhie, Wang, Liu, Warren, Storey, Espley and Allan. This is an open-access article distributed under the terms of the Creative Commons Attribution License (CC BY). The use, distribution or reproduction in other forums is permitted, provided the original author(s) or licensor are credited and that the original publication in this journal is cited, in accordance with accepted academic practice. No use, distribution or reproduction is permitted which does not comply with these terms. 